\title{
Abdeckung des ovalen/ \\ runden Fensters mit und ohne \\ kortisongetränkten autologen \\ Bindegewebspartikeln im Rahmen \\ der Hörsturzbehandlung
}

\subsection{Einleitung}

\subsubsection{Hinführung zum Thema}

Die neueste Entwicklung bei der operativen Behandlung der idiopathischen, akuten, sensorineuralen Schwerhörigkeit (ISSNHL) stellt die im Rahmen einer explorativen Tympanoskopie gleichzeitig vorgenommene Obliteration der runden und ovalen Fensternischen mit autologem Bindgewebe dar, das zu Beginn des chirurgischen Eingriffs entnommen und bis zum Einbringen in die Pauke in einer kortisonhaltigen Lösung getränkt wurde [31, 52, 53]. Damit kombiniert dieses Vorgehen gewissermaßen die Effekte der explorativen Tympanoskopie mit Abdeckung der häutigen Membran/en, die den Innenohr- vom Mittelohrraum abtrennt/en, und die einer Applikation von Glukokortikoiden in das Tympanon, wie sie ansonsten bei den intratympanalen Kortikoidinjektionen/-infusionen vorgenommen wird.

\subsubsection{Fragestellung}

Im vorliegenden Kapitel werden die nach unseren Recherchen ersten Ergebnisse, die mit der Methode der Implantation kortisongetränkten autologen Bindegewebes an Patientenkollektiven gesammelt wurden, soweit es geht, mit den Resultaten der explorativen Tympanoskopie unser eigenen Studiengruppe verglichen, bei der das in die Pauke verbrachte Bindegewebe vorher nicht mit Kortison in Kontakt gekommen war. 


\subsection{Ergebnisse}

\subsubsection{Studien mit explorativer Tympanoskopie und Abdichtung des runden/ovalen Fensters mit kortisongetränktem autologem Binde-/Fettgewebe}

\subsubsection{Charakterisierung der Studien}

Wir konnten in der Literatur 3 Studien finden, in denen bei Patientenkollektiven kortisongetränktes Bindegewebe in die runde/ovale Fensternische verbracht worden war (Tab. 8.1) [31, 52, 53]. Dabei stammten zwei Arbeiten von demselben Erstautor [52, 53], sie wurden in 4-jährigem Abstand voneinander veröffentlicht.

Beide Publikationen von Loaderer et al. (2013 und 2017) verwendeten zur Ermittlung der Hörverluste/Hörgewinne einen Mittelwert, der aus den Tonfrequenzen $0,5 \mathrm{kHz}, 1 \mathrm{kHz}, 2 \mathrm{kHz}$ und $3 \mathrm{kHz}\left(\mathrm{PTA}_{4}\right)$ gebildet wurde. Ferner war als Filterkriterium festgesetzt worden, dass die durch den Hörsturz ausgelösten Hörverluste mindestens $50 \mathrm{~dB}$ zu betragen hatten [52, 53]. Als Glukokortikoid war Triamcinolon gewählt worden, wobei $40 \mathrm{mg}$ in $1 \mathrm{ml}$ Suspensionsmittel aufgelöst worden war. Zwischen dem stationären Aufnahmetermin und dem Operationstag waren die Patienten systemisch intravenös mit Steroiden (Prednisolon 500 mg i.v.) für mindestens 3 Tage behandelt worden.

In der Veröffentlichung von Loader et al. von 2013 wurden die Hörgewinne nach den modifizierten Kanzaki-Kriterien angegeben [53]. Daneben wurde, ohne den Anfangs- und Endhörverlust zu benennen, der Hörgewinn in dem Patientengut in Dezibel mitgeteilt.

Tab. 8.1 Beschreibung der Studien, bei denen Cortison getränktes Bindgewebe bei der Hörsturzbehandlung im Rahmen einer Tympanoskopie in die runde und ovale Fensternische verbrachten worden waren

\begin{tabular}{l|l|l|l|l|l|l|l|l}
\hline Studie & $\mathrm{n}$ & $\begin{array}{l}\text { prä- } \\
\text { OP }\end{array}$ & $\begin{array}{l}\text { post- } \\
\text { OP1 }\end{array}$ & HG1 & $\begin{array}{l}\text { post- } \\
\text { OP2 }\end{array}$ & HG2 & $\begin{array}{l}\text { Kanzaki } \\
1+2\end{array}$ & $\begin{array}{l}\text { Kanzaki } \\
1-3\end{array}$ \\
\hline Eigene (ohne Cortison) & 36 & 99,3 & 97,2 & 2,1 & & & 16,1 & 32,3 \\
\hline $\begin{array}{l}\text { Loader 2017 (ohne } \\
\text { Cortison) }\end{array}$ & 28 & 76 & 58 & 18 & & & & 63 \\
\hline $\begin{array}{l}\text { Loader 2017 (mit } \\
\text { Cortison) }\end{array}$ & 25 & 73 & 42 & 31 & & & & 83 \\
\hline $\begin{array}{l}\text { Loader 13 (mit Cortison) } \\
\text { Eigene (ohne Cortison) }\end{array}$ & 13 & 97,5 & & & 86,3 & 20 & 30,8 & 61,6 \\
\hline Hofmann (mit Cortison) & 54 & 113 & 98 & 15 & 86,7 & 26 & 45 & 69 \\
\hline
\end{tabular}

$\mathrm{n}=$ Anzahl der Patienten; prä-OP = präoperativer Wert; post-OP1 = posttherapeutischer Wert 1.-5. Tag in $\mathrm{dB}$; $\mathrm{HG} 1=$ Hörgewinn in $\mathrm{dB}$; post-OP2= posttherapeutischer Wert mit minimal 9 Tagen Abstand zur OP in dB; Kanzaki $1+2=$ Patienten, die der Hörerholung nach den modifizierten „Kanzaki“"kriterien 1 und 2 entsprachen ( $>30 \mathrm{~dB}$ Hörgewinn); Kanzaki 1-3= Patienten, die der Hörerholung nach den modifizierten „Kanzaki“-kriterien 1-3 entsprachen (>10 dB Hörgewinn) 
In der späteren Veröffentlichung von Loader et al. (2017) wurde über zwei Patientengruppen berichtet, die parallel zueinander mittels explorativer Tympanoskopie sowie Abdichtung des runden und ovalen Fensters behandelt worden waren [52]. In der einen Gruppe war das implantierte körpereigene Fasziengewebe kortisongetränkt, in der anderen Patientengruppe nicht. In dieser Studie wurden die durchschnittlichen Anfangshörverluste, Endhörverluste (1.5. postoperativer Tag) und die Hörgewinne jeweils in Dezibel angegeben. Bei den Patienten, in deren Pauke kortisongetränktes Bindegewebe verbracht worden war, betrug die Latenzzeit zwischen Hörsturzereignis und Tympanoskopietermin 9 Tage, bei den Patienten, die kein vorbehandeltes Bindegewebe erhalten hatten, lag dieser Zeitraum bei 13 Tagen.

Inwiefern es Überschneidungen in der Aufnahme einzelner/aller Patienten in diesen beiden Studien gibt, ist nicht gesondert vermerkt. Dies würde aber für unsere Betrachtungen im Wesentlichen unerheblich sein, da in der früheren Studie die Therapiebewertung aufgrund der modifizierten Kanzaki-Kriterien erfolgte, die spätere Veröffentlichung die Hördaten in Dezibelwerten in den Mittelpunkt der Betrachtung nahm und damit keine „Ergebnisüberschneidungen“ möglich waren.

In der jüngst veröffentlichten Arbeit von Hofmann et al. (2020) war als Ausgangskriterium für die Durchführung der explorativen Tympanoskopie ein initialer Hörverlust von $60 \mathrm{~dB}$ gewählt worden [31]. Der durchschnittliche Hörverlust wurde anhand der Hörschwellenwerte bei den Frequenzen 0,5, 1, 2 und $4 \mathrm{kHz}$ bestimmt. Hofmann et al. bezogen sich in ihren Hörverlustangaben auf Medianwerte, nicht auf das arithmetische Mittel. Zu Beginn der Operation entnommenes Faszien- oder Fettgewebe wurde in Dexamethasonphosphat getränkt, bevor es in die runde/ovale Fensternische verbracht wurde. Die mediane Latenzzeit zwischen dem Hörsturzereignis und dem chirurgischen Eingriff betrug 5 Tage. Nachuntersuchungen zur Beurteilung der Therapieergebnisse wurden am 21. Tag nach der Operation (Zeitpunkt der Detamponierung) und nach 3-6 Monaten durchgeführt. Ein Schwerpunkt der Arbeit besteht darin, dass Unterscheidungen nachgegangen wurde, die klären sollten, ob sich das Einbringen von kortisongetränktem Bindegewebe 1) allein in die runde Fensternische, 2) allein in die ovale Fensternisch, 3) in beide Fensternischen zusammen auf die Therapieerfolge ausgewirkt hat. Auch die in dieser Arbeit ausgewerteten Patienten hatten initial nach der Krankenhausaufnahme eine systemische Hochdosissteroidtherapie erhalten (Prednisolon $250 \mathrm{mg}$ i.v.).

\subsubsection{Rekrutierung des Patientengutes}

Die Charakterisierung der Studien weist darauf hin, dass unterschiedliche Filterkriterien bei der Auswahl des Patientengutes, die für eine explorative Tympanoskopie vorgesehen wurden, und unterschiedlich Auswertungsverfahren zur Bestimmung der Therapieeffekte bestanden. Dies führt dazu, dass die Studien von Loader et al. auf der einen und die von Hofmann et al. auf der anderen Seite nicht untereinander vergleichbar sind. Um die Behandlungsresultate der hier vorgestellten Studien den Ergebnissen unseres Patientengutes gegenüberzustellen, 
mussten wir die Daten der von uns behandelten Patienten entsprechend den Auswertungsvorgaben der genannten Autoren anpassen.

Dies bedeutete, dass für den Vergleich mit den Studien von Loader et al. zunächst diejenigen Patienten aus unserem Gesamtkrankengut zusammengefasst werden mussten, deren gemittelte Latenzzeit zwischen Hörsturzereignis und Tympanoskopietermin 9 Tage betrug. Ferner hatten wir die gemittelten Hörverluste aus den 4 Frequenzen $0,5,1,2$ und $3 \mathrm{kHz}$ zu bilden. Schließlich bewerteten wir nur den jeweils zeitlich gesehen letzten Hörwert, der innerhalb der ersten 5 Tage nach der Operation bei den Patienten gemessen worden war. Eine Anpassung unserer Patienten an das Aufnahmekriterium, dass Patienten mit anfänglichen Hörverlusten auch unter $60 \mathrm{~dB}$ bis hin zu $50 \mathrm{~dB}$ in die Auswertung aufgenommen werden konnten, war im Nachhinein nicht mehr zu realisieren.

Für den Vergleich mit der Arbeit von Hofmann et al. haben wir aus den gesammelten Hörverlusten unseres Krankengutes ebenfalls die Medianwerte bestimmt. Den Patienten, deren erste postoperative Tonschwellenaudiometrie - wie bei Hofmann et al. - am 21. Tag nach der Tympanoskopie lag, konnten wir keine Vergleichspopulation gegenüberstellen. Die Werte, die im 3.-6. Monat nach dem chirurgischen Eingriff erfasst worden waren, haben wir mit unseren Hördaten, die im Rahmen der Nachuntersuchung erfasst wurden, vergleichen können, indem wir uns auf ein vergleichbares Zeitintervall in der Followup-Beobachtung, wie es bei Hofmann et al. vorgegeben war, bezogen.

\subsubsection{Vergleich der Hörerfolge nach Tympanoskopie mithilfe der Medianwerte der Anfangs- und Endhörverluste}

\subsubsection{Mediane Hörverluste vor und nach der Tympanoskopie in unserem Krankengut}

Der mediane initiale Hörverlust bei den aus unserem Gesamtkrankengut „herausgefilterten" 13 Patienten, die in ihren Rahmenbedingungen denen aus der Publikation von Hofmann et al. entsprachen, betrug 107,5 dB $(n=7)(A b b .8 .1)$. Nach durchschnittlich 127 Tagen lag der Endhörverlust im Median bei 76,3 dB, es war zu einer medianen Hörverbesserung im Mittel von 31,2 dB gekommen.

\subsubsection{Krankengut von Hofmann et al.}

Die Vergleichswerte von Hofmann et al. betrugen in der Gruppe der Patienten, bei denen beide Fensternischen mit kortisongetränktem Eigengewebe obliteriert worden waren, am Anfang $113 \mathrm{~dB}$ (Medianwert; $\mathrm{n}=54$ ) und nach 3-6 Monaten 86,7 dB. Hier war ein durchschnittlicher medianer Hörgewinn von 26,3 dB eingetreten.

\subsubsection{Vergleichende Betrachtung der untersuchten Patientenkollektive}

Man erkennt, dass sich, von ähnlichen Anfangswerten ausgehend, am Ende der unterschiedlichen Nachbeobachtungszeiten Endhörverluste eingestellt hatten, die 


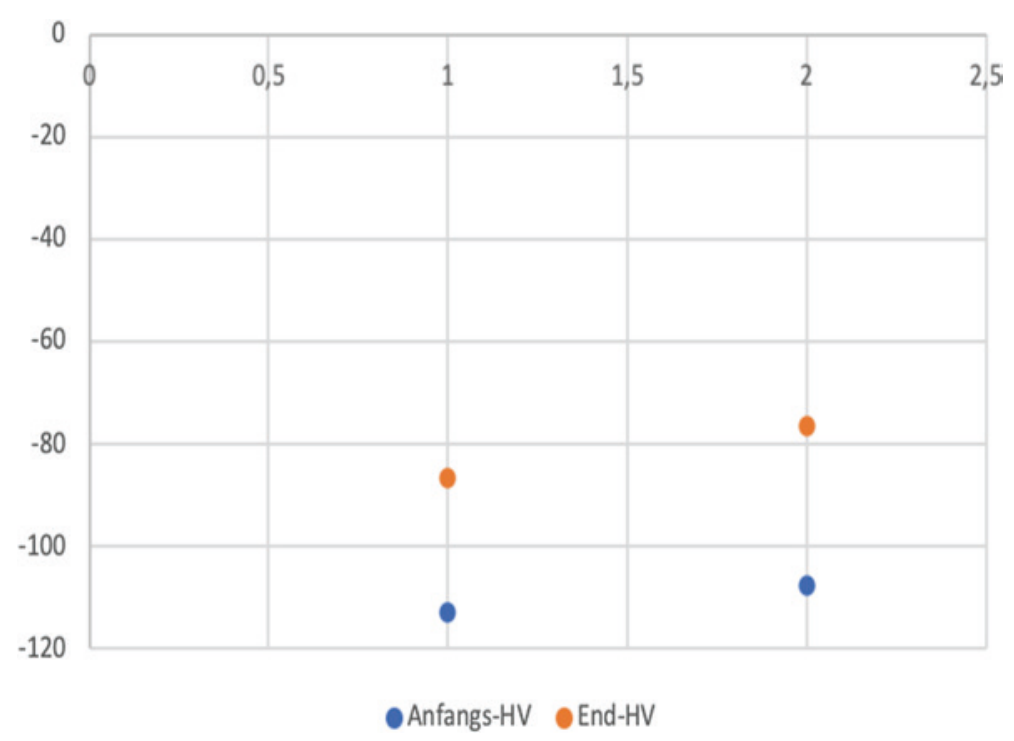

Abb. 8.1 Mediane Hörentwicklung bis zum 3.-4. Monat nach Tympanoskopie mit Abdeckung der runden und ovalen Fensternische. Abszisse: Studiengruppen: linkes vertikales Punktepaar: mediane Hörverluste vor und nach der Einbringung kortisongetränkten Bindegewebes (Hofmann et al., $\mathrm{n}=54$ ); rechtes vertikales Punktepaar: mediane Hörverluste vor und nach der Einbringung von unbehandeltem Bindegewebe (Eichhorn et al.; $\mathrm{n}=31$ ). Ordinate: Mediane Hörverlustwerte in $\mathrm{dB}$; unterer Bildpunkt: Anfangshörverlust; oberer Bildpunkt: Resthörverlust bei der Nachuntersuchung

sich bei Betrachtung der Medianwerte um weniger als $10 \mathrm{~dB}$ unterschieden. Dabei lag der mediane Hörgewinn in der Gruppe der Patienten, bei der die Fensternischen mit kortisonhaltigem Bindgewebe ausgefüllt worden war, im Median um 4,9 dB geringer als bei Patienten, deren Obliteration der Fensternischen ohne Glukokortikoid-getränktes Bindegewebe erfolgt war.

Interessant erscheint in diesem Zusammenhang die Tatsache, dass die mediane Hörerholung, die Hofmann et al. bei ihren Patienten nach der 3. postoperativen Woche abgeleitet hatten, erst $57 \%(15 \mathrm{~dB})$ des maximalen Hörgewinnes $(26,3 \mathrm{~dB})$ entsprach, den die Erkrankten nach 3-6 Monaten erreicht hatten. Dies widerspricht unserer in Kap. 3 ausführlich beschriebenen Erfahrung.

\subsubsection{Vergleich der Hörerfolge nach Tympanoskopie innerhalb der ersten fünf postoperativen Tage}

\subsubsection{Hörverlustwerte vor der OP und innerhalb der ersten 5 postoperativen Tage}

Für den Vergleich der Behandlungswirkung mit den beiden Arbeiten von Loader et al. haben wir in unserem Patientengut die mittleren Hörverluste aus den Frequenzen 0,5, 1, 2 und $3 \mathrm{kHz}$ gebildet [52,53]. Darüber hinaus haben wir von 
unserem Kollektiv nur jene operierten Patienten in die Datenanalyse einbezogen, die Hörverluste innerhalb der ersten 5 Tage nach der Operation erzielt hatten $(\mathrm{n}=32)$.

\subsubsection{Eigenes Krankengut}

Der initiale Hörverlust bei diesem gefilterten Krankengut betrug 99,3 dB (SAW: 17,1 dB; $\mathrm{n}=32$ ) (Abb. 8.2). Im Mittel 2,9 Tage nach der OP (Variationsbreite: 1-5 Tage) lag die Hörminderung noch bei 97,2 dB (SAW: 26,7 dB; $n=32$ ) (Abb. 9.2). Dies bedeutet, dass die Patienten in dieser kurzen Zeit nach der Operation einen Hörgewinn von durchschnittlich 2,1 dB erreicht hatten. Der Hörverlust zum letzten Nachuntersuchungszeitpunkt (2,3 Jahre nach der Operation) lag dann bei 69,4 dB (SAW: 31,4 dB; $n=32$ ), was einer Hörerholung von 29,9 dB entsprach.

\subsubsection{Patientengut von Loader et al. mit kortisongetränktem Bindegewebe}

In dem Patientengut von Loader et al. (2017) betrug der Ausgangshörverlust bei den Erkrankten, denen mit Kortison getränktes Bindegewebe implantiert worden war, $73 \mathrm{~dB} \quad(\mathrm{n}=25)$ (Abb. 8.2) [53]. Innerhalb der ersten 5 Tage nach dem chirurgischen Eingriff wurde mit einem Hörgewinn von $31 \mathrm{~dB}$ ein „Endhörverlust“" von 42 dB erzielt. In einer früher veröffentlichten Studie (2013) berichteten Loader et al. bei identischen Auswertungskriterien über eine geringere Hörverbesserung von nur 20,4 dB.

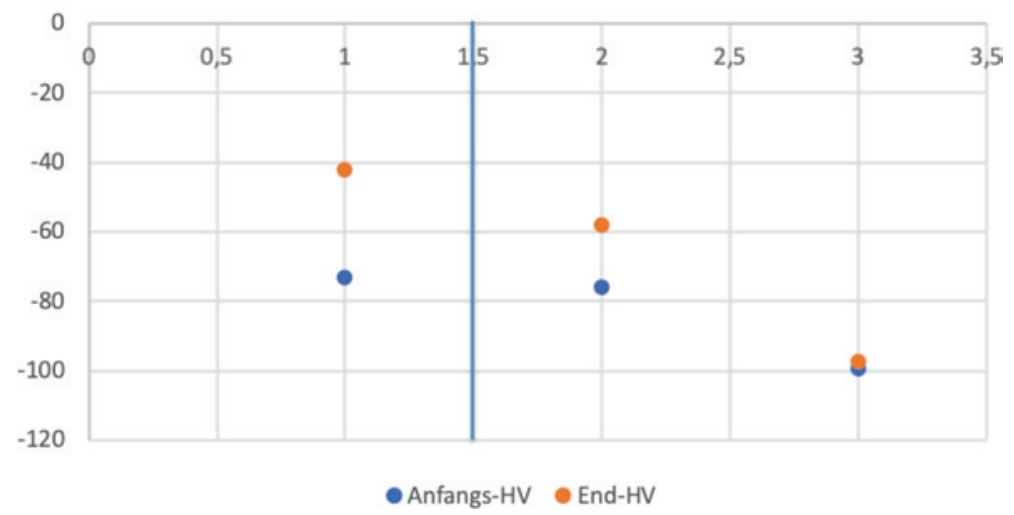

\footnotetext{
Abb. 8.2 Gemittelte Hörentwicklung nach Tympanoskopie innerhalb der ersten 5 postoperativen Tage. Abszisse: linkes vertikales Punktepaar: Einbringung von kortisongetränktem Bindegewebe in die Fensternischen (Loader et al. 2017; $\mathrm{n}=25$ ); mittleres vertikales Punktepaar: Einbringung von unbehandeltem Bindegewebe in die Fensternischen (Loader et al. 2017; $\mathrm{n}=28$ ); rechtes vertikales Punktepaar: Einbringung von unbehandeltem Bindgewebe in die Fensternischen (eigene Studie; $\mathrm{n}=7$ ); Ordinate: Hörverlustwerte in $\mathrm{dB}$; unterer Bildpunkt: Anfangshörverlust; oberer Bildpunkt: Resthörverlust bei der Nachuntersuchung
} 


\subsubsection{Patientengut von Loader et al. mit unbehandeltem Bindgewebe}

Die Publikation von Loader et al. aus dem Jahr 2017 beinhaltete auch eine Vergleichspopulation von Patienten, bei der im Rahmen der Tympanoskopie unbehandeltes Bindegewebe - wie bei unseren Erkrankten - in beide Fensternischen eingebracht worden war (Abb. 8.2) [53]. Hier lag der Anfangshörverlust bei $76 \mathrm{~dB}$ und der „Endhörverlust" bei $58 \mathrm{~dB}$. Dem entsprach eine Hörverbesserung von $18 \mathrm{~dB}$.

\subsubsection{Vergleichende Betrachtung der untersuchten Patientenkollektive}

Wie bereits in Kap. 3 beschrieben, traten in unserem Patientengut deutlich sichtbare Hörerholungen erst ab dem 10. postoperativen Tag auf. Damit unterscheiden wir uns erheblich von den Ergebnissen, die Loader et al. sowohl bei der Verwendung von kortisongetränktem als auch nicht vorbehandeltem körpereigenem Gewebe erreicht hatten. Leider liegen aus diesen beiden Patientenkollektiven keine zu einem späteren Zeitpunkt erhobenen Hörwerte vor, die hätten anzeigen können, ob es in der Folgezeit nach dem 5. postoperativen Tag noch zu weiteren Hörverbesserungen gekommen war. In unserem Krankengut betrug der relative Hörgewinn mit 2,1 dB nur 6,5 \% von der gesamten Hörerholung, die nach 2,3 Jahren durchschnittlich 32,5 dB entsprach. Auch die Arbeit von Hofmann et al. zeigte, dass selbst 3 Wochen nach der Tympanoskopie mit einer Hörverbesserung von $15 \mathrm{~dB}$ erst 63,6\% des insgesamt erreichbaren Hörerfolges (26,3 dB) eingetreten war, der sich 3-6 Monate nach dem chirurgischen Eingriff messen ließ.

Umgekehrt steht die Beobachtung von Hofmann et al. aber auch im Widerspruch zu unseren Ergebnissen, nach denen der endgültig verbleibende Hörgewinn bereits nach etwa 3 Wochen eingetreten ist (s. Kap. 3).

Die Abb. 8.3 zeigt deutlich, wie unterschiedlich sich die postoperative Hörentwicklung in den einzelnen Studiengruppen darstellt. Dabei sind die prozentualen Hörverluste in Abhängigkeit von der anfänglich bestehenden, präoperativen Hörminderung (=100\% Hörverlust) aufgetragen. Die Bezugssysteme zwischen den Studien variieren, indem Hofmann et al. Medianwerte angegeben hatten und bei Loader et al. und unserer Studie die mittleren PTA 4 -Werte den Auswertungsberechnungen zugrunde gelegt waren. Es zeigt sich ein sehr ähnliche zeitliche Kurvenverlauf in den Patientenkollektiven von Hofmann et al. und unserer Studie, während bei Loader et al. jeweils die sehr rasch nach der Operation einsetzenden Hörverbesserungen $\mathrm{zu}$ beobachten sind, die weder Hofmann et al. noch wir bei unseren Patienten beobachten konnten. Darüber hinaus werden bei Loader et al. (2017) in der Gruppe der mit kortisongetränktem Bindgewebe Behandelten die besten Hörresultate (57,5\%), bezogen auf den Ausgangshörverlust erreicht. Die prozentualen Hörerholungen bei den Patienten von Hofmann et al. (73,3\%) zeigten etwa dieselbe Größenordnung, wie sie bei Loader et al. (76,3\%) und unseren Patienten $(79,5 \%)$ erreicht wurden, die kein vorbehandeltes autologes Gewebe implantiert bekommen hatten. 


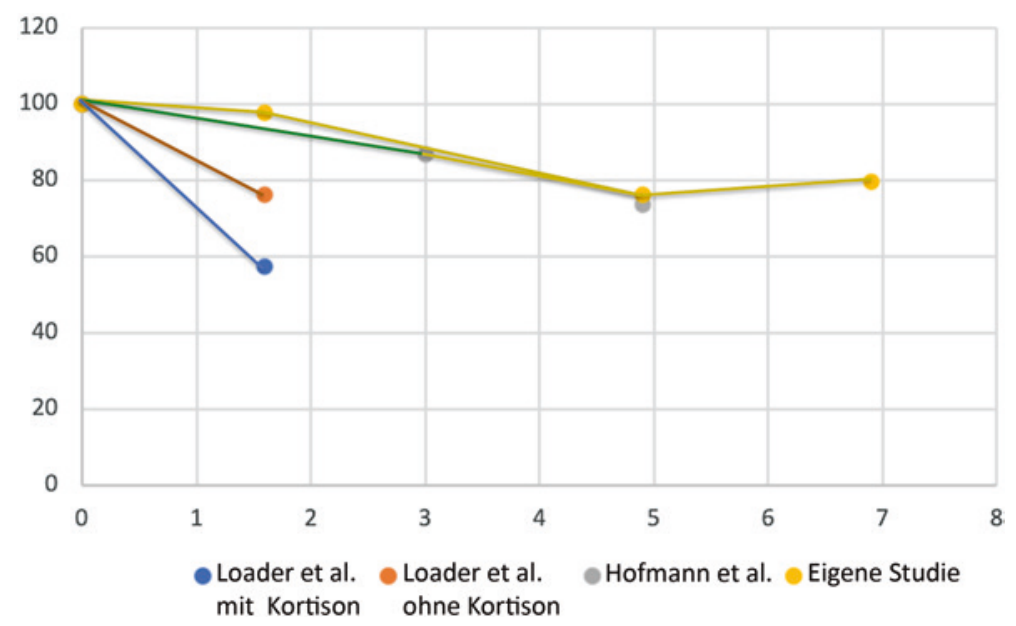

Abb. 8.3 Zeitverlauf der Hörerholung nach der Tympanoskopie; Abszisse: Zeitlicher Abstand nach der Tympanoskopie in Tagen; Abszisse: Zeitpunkt nach der Tympanoskopie; Ordinate: Prozentuale mediane (Hofmann et al.) und prozentuale arithmetisch gemittelte Hörverluste (Loader et al. und Eichhorn) bezogen auf den Anfangshörverlust, Angabe in Wochen nach der Operation;

\subsubsection{Vergleich der Hörgewinne auf der Basis der modifizierten Kanzaki-Kriterien}

Bei dem Vergleich der Hörgewinne auf der Basis der modifizierten Kanzaki-Kriterien können wir den bei unseren Patienten erhobenen Daten drei Studiengruppen gegenüberstellen, wenngleich die Nachuntersuchungstermine sehr unterschiedlich gewählt wurden, was die Vergleichbarkeit insbesondere der Ergebnisse von Loader et al. mit denen von Hofmann et al. wie auch unserem Patientenkollektiv infrage stellt.

Bei der Verwendung kortisongetränkten Bindegewebes wurden, bezogen auf die modifizierten Kanzaki-Typen 1 und 2, Hörerholungsraten von $>30 \mathrm{~dB}$ zwischen $32 \%$ (Loader et al. 2013) und $45 \%$ (Hofmann et al.) erreicht (Abb 8.4) $[31,52]$. Bezieht man alle Hörgewinne von $>10 \mathrm{~dB}$ (Kanzaki-Typ 1-3) in die Auswertung ein, konnten Hörverbesserungen zwischen $52 \%$ (Loader et al. 2013) und $83 \%$ (Loader et al. 2017) erreicht werden [52, 53]. Berechnet man aus den zwei Studien von Loader et al. 2013 und 2017 den Mittelwert, betrug der Hörgewinn bei diesem zusammengefassten Patientenkollektiven 67,5\%.

Loader et al. (2017) hatten auch eine Studiengruppe mitgeführt, in der das in die Pauke eingebrachte Bindegewebe nicht zuvor in Kortison getränkt war [53]. Diese Patienten erreichten bis zum 5. postoperativen Tag in $63 \%$ der Fälle Hörerholungen von $>10 \mathrm{~dB}$. Im Vergleich dazu betrug der Wert in unserem Krankengut nur 32,3\%.

Im Vergleich zum Patientenkollektiv von Loader et al. fällt bei den Patienten unserer Studie, bei denen kortisongetränktes oder auch nicht behandeltes Bindegewebe Verwendung fand, auf, dass sie innerhalb der ersten 5 Tage nach Tympanoskopie nur etwa halb so hohe Hörerholungsraten vorweisen konnten, wie sie Loader et al. von ihrem Krankengut berichteten. Damit lagen wir aber bereits 


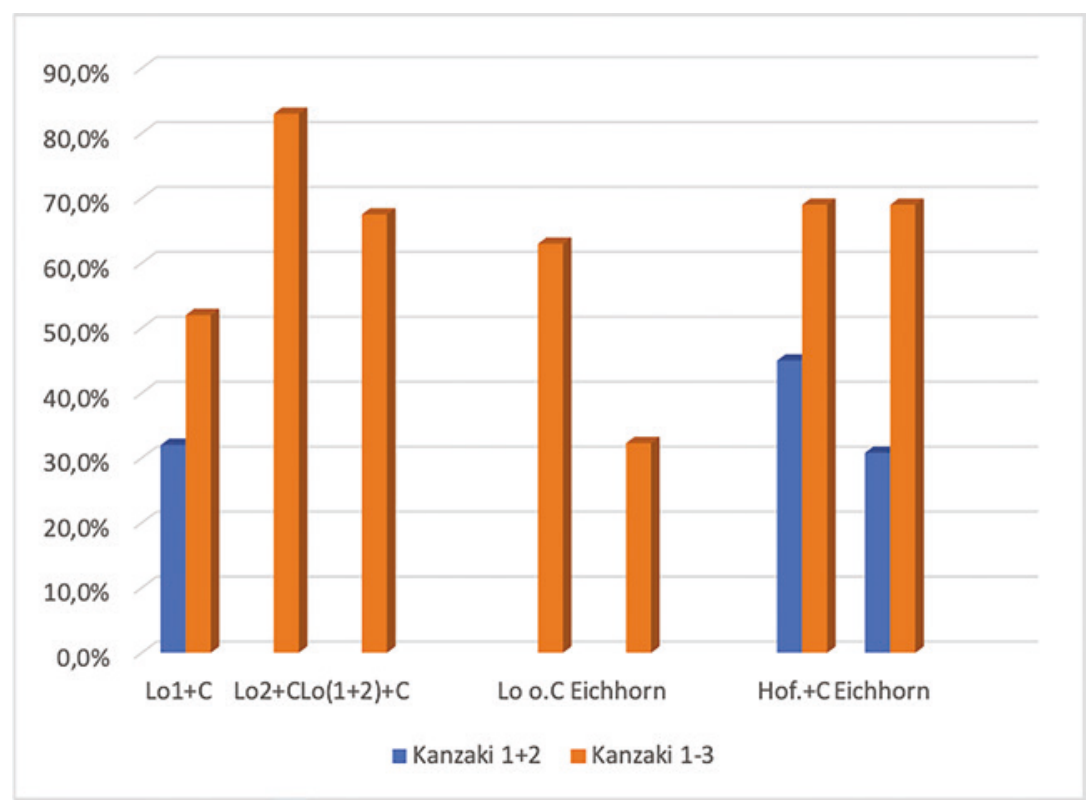

\begin{abstract}
Abb. 8.4 Hörerholung nach den modifizierten Kanzaki-Kriterien bei Patienten mit und ohne Kortison-getränkten Bindgewebeobliterationen der runden und ovalen Fensternischen. Blaue Säulen: Hörerholung nach den mod. Kanzaki-Typen 1 und 2 ( $>30 \mathrm{~dB}$ Hörerholung); rote Säulen: Hörerholung nach den mod. Kanzaki-Typen 1-3 (>10 dB Hörerholung). Abszisse: Studiengruppen; $1=$ Loader et al. 2013; $2=$ Loader et al. 2017;3=zusammengefasste Werte aus Loader et al. 2013 und 2017; 5=Loader et al. ohne Kortison (2017; innerhalb von 5 Tagen nach Tympanoskopie); $5=$ eigene Studie ohne Kortison (innerhalb von 5 Tagen nach der Tympanoskopie); 8=Hofmann et al. mit Kortison (3-6 Monate nach Tympanoskopie); 9=eigene Studie ohne Kortison (3-6 Monate nach der Tympanoskopie); Ordinate: Anteil der Patienten, die die mod. Kanzaki-Kriterien Typ 1-3 erfüllten (in Prozent)
\end{abstract}

deutlich höher, als es der Vergleich der Hörwerte auf der Basis der initialen und Endhörverluste erkennen ließ.

Bezogen auf die Hörverbesserungen der in der Studie von Hofmann et al. vorgelegten Daten 3-6 Monate nach dem chirurgischen Eingriff erreichten unsere Patienten bei den Kanzaki-Typen 1-3 gleich gute Resultate (beide Studienkollektive $69 \%$ ).

\title{
8.2.5 Vergleich des Zeitverlaufes der Hörerholung bei Patienten nach intratympanaler Steroidtherapie und Tympanoskopie
}

\subsubsection{Problemstellung}

Die Diskrepanz der Ergebnisse bezogen auf den Zeitverlauf der Hörerholung, insbesondere zwischen Loader et al. auf der einen und Hofmann et al. sowie unseren Resultaten auf der anderen Seite, veranlasste uns dazu, auch die Gruppe 
der Patienten, die intratympanale Kortikoidinjektionen erhielten (s. Kap. 9), mit unserem Patientengut - Tympanoskopie mit Einbringen von unbehandeltem Bindgewebe - bezüglich des zeitlichen Ablaufes der Hörrestitution zu vergleichen. Dabei sollten Anhaltspunkte dafür gewonnen werden, wie intratympanal eingebrachtes Kortison seine Wirkung auf die Innenohrhörfunktion entfalten kann.

\subsubsection{Rekrutierung des Patientengutes}

Aus publizierten Studien, die entweder intratympanale Kortikoidinjektionen als Einzeltherapie, zeitlich parallel zu systemischen Steroidinjektionen oder sequenziell nach einer systemischen Kortikoidbehandlung anwendeten, konnten wir 12 Patientengruppen extrahieren, bei denen sich im zeitlichen Abstand zum Therapieabschluss mehr als eine Angabe zu den verbliebenen Hörverlusten finden ließen [36, 40, 46, 77, 85, 90, 95, 100]. Das Kollektiv, auf dass wir insgesamt zurückgreifen konnten, betrug dabei 436 Patienten. Dem stellten wir den Zeitverlauf von 31 tympanoskopierten Patienten aus unserer Studie gegenüber.

\subsubsection{Zeitlicher Ablauf der Hörerholung bei Patienten mit intratympanalen Kortikoidinjektionen und explorativen Tympanoskopien}

Wenn man den relativen verbliebenen Hörverlust bezogen auf die initiale Höreinschränkung $(=100 \%) \quad \mathrm{zu}$ einzelnen Zeitpunkten nach Abschluss der intratympanalen Steroidinjektionen bzw. dem Operationsdatum aufträgt, erhält man die in Abb. 8.5 abgebildeten Kurven. Dabei sieht man, dass die Patienten zum Ende der in der Regel 14-tägigen intratympanalen Steroidtherapie im gewichteten Mittel sehr schnell Hörerfolge erreicht hatten, die einem prozentualen Hörgewinn von 28,7\% entsprachen und sich in der Folgezeit bis zu einem 1/2 Jahr nach der Krankenhausbehandlung kaum noch steigern ließen (Endwert: 34,3\%). Demgegenüber stieg bei den Tympanoskopierten der Hörverlust unmittelbar nach der Operation (1. und 2. postoperativer Tag) sogar erst einmal um weitere 6,1\% an. Erst dann setzt eine nahezu lineare Hörverbesserung bis zum 15. Tag nach dem chirurgischen Eingriff ein, der dann insgesamt 33,5\% vom Ausgangswert ausmachte. In der Folgezeit kam es nur noch zu marginalen weiteren Veränderungen der Hörleistung.

\subsubsection{Einordnung der Studienergebnisse von Loader et al. und Hofmann et al.}

Der zeitliche Ablauf der Hörerholung in der Studie von Loader et al. spiegelt also eher den Verlauf wieder, der sich bei Patienten mit intratympanalen Kortikoidinjektionen zeigt, während er in der Studie von Hofmann et al. dem einer etwas verzögert einsetzenden Hörverbesserung entspricht, die auch wir beobachtet haben. Sollten die Resultate von Loader et al. eher den Tatsachen entsprechen, als die von Hofmann et al., würde dies bedeuten, dass die Kortikoidwirkung an den beiden Fenstermembranen zu einem sehr raschen Hörerfolg führt, der dem der Tympanoskopie innewohnenden Trend, nämlich in den ersten Tagen nach 


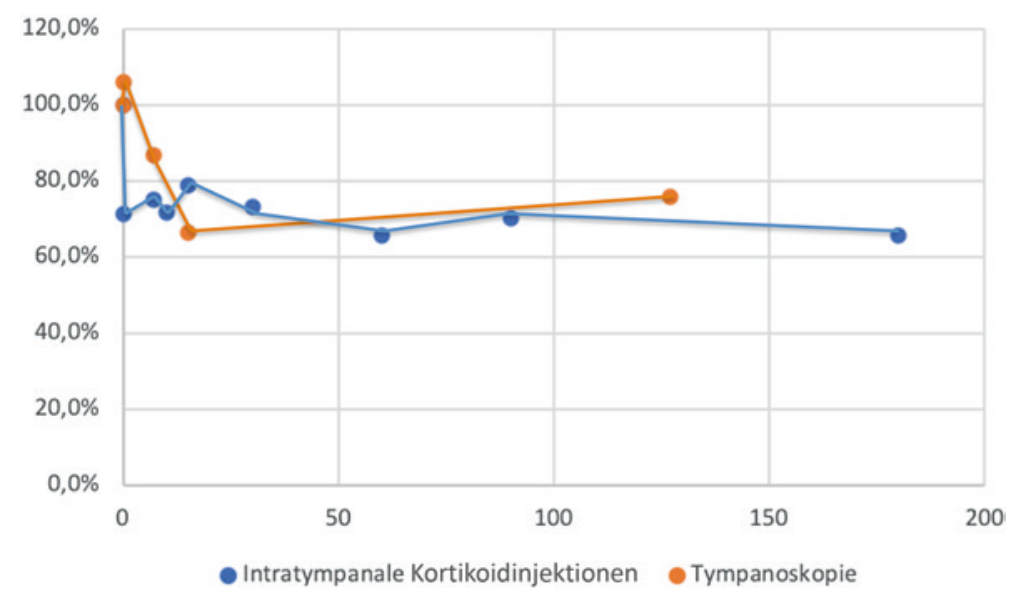

Abb. 8.5 Zeitverlauf der prozentualen Hörentwicklung bei Patienten mit intratympanalen Kortikoidinjektionen $(\mathrm{n}=436$; blaue Kurve $)$ und Tympanoskopie $(\mathrm{n}=31$; rote Kurve). Abszisse: Tage nach der Therapie; Ordinate: Hörgewinn im Vergleich zum Ausgangswert $(=100 \%)$ in Prozent

dem Eingriff erst einmal keine Hörverbesserungen erzielen zu können, anfangs übertrifft. Weitere Untersuchungen sollten zur endgültigen Erhellung dieses Problemkomplexes durchgeführt werden. Dabei muss bedacht werden, dass der aktuelle Vergleich des Einbringens kortisongetränkten Bindegewebes vs. intratympanaler Kortikoidinjektionen dahin gehend beeinträchtigt ist, dass die erste Hörmessung nach ITST-Behandlung erst 14 Tage nach der ersten Medikamenteninjektion in die Pauke erfolgt war und damit der Wirkungseintritt des Pharmakons schon einige Zeit zurücklag, während der Wirkungseintritt bei der Implantation von kortisongetränktem Bindgewebe unmittelbar in den darauffolgenden Tagen von Loader et al. beobachtet worden war.

Würde man umgekehrt die Ergebnisse von Hofmann et al. als real ansehen, wäre die Wirkung auf die Hörverbesserung hauptsächlich von dem operativen Eingriff ausgegangen und hätte auch dessen zeitlichen Wirkungsmuster entsprochen. Hier wäre der Effekt des Kortisons auf die Hörverbesserung deutlich in den Hintergrund getreten.

Die vorliegenden Daten aus 3 Studien bilden eine noch ungenügende Grundlage, um die Therapieeffekte der explorativen Tympanoskopie mit Einbringen von kortisongetränktem autologen Bindegewebe abschließend beurteilen und mit andere Therapieverfahren, die auch beim Hörsturz eingesetzt werden, vergleichen zu können. Unterschiedliche Auswertungskriterien erschweren bis heute zusätzlich eine Gegenüberstellung der einzelnen Studienergebnisse. Es bleibt die Frage offen, ob es bei der Einbringung von kortisongetränktem Bindegewebe in die Pauke unmittelbar nach dem Eingriff zu einer Hörverbesserung kommt oder ob die Hörverbesserung, wie bei der Tympanoskopie mit Obliteration der Fensternischen mit nicht vorbehandeltem Bindgewebe, erst verzögert einsetzt. 


\subsection{Zusammenfassung}

Bislang liegen nach unseren Recherchen erst drei Studien vor, in denen bei Patienten mit ISSNHL kortisongetränktes Bindegewebe in die runden und ovalen Fensternischen verbracht wurden. In diesem Kapitel werden die Hörerholungen der auf diese Weise behandelten Patienten mit den Patienten verglichen, bei denen kein vorbehandeltes Bindegewebe verwendet wurde. Darüber hinaus werden die Zeitabläufe der Hörentwicklung bei beiden Therapiearten untersucht und den Hörerholungen gegenübergestellt, die bei intratympanalen Kortikoidinjektionen erreicht wurden.

Die zur Verfügung stehenden Studien haben sehr unterschiedliche Auswertungskriterien (mediane vs. arithmetisch gemittelte Hörverluste; Nachbeobachtungszeit von 5 Tagen nach Tympanoskopie vs. 90-180 Tagen; PTA-Bestimmung aus den Frequenzen $0,5,1,2$ und $3 \mathrm{kHz}$ vs. 0,5, 1, 2 und $4 \mathrm{kHz}$ ) gewählt, so dass eine vergleichende Betrachtung der mit kortisongetränktem Bindegewebe behandelten Patienten untereinander nicht möglich war.

Bei langfristigen Nachbeobachtungen zwischen 90 und 180 Tagen wurden bei Obliteration der runden und ovalen Fensternischen mit Steroid-getränktem autologem Bindgewebe mediane Hörverbesserungen von 26,3 dB zu einem medianen Endhörverlust von $86,7 \mathrm{~dB}$ erreicht $(n=58)$. Dem stand in der Gruppe der Erkrankten, bei denen kein vorbehandeltes Bindegewebe verwendet wurde, eine Hörerholung von 31,2 dB auf einen medianen Endhörverlust von 76,3 dB gegenüber $(n=7)$.

Innerhalb der ersten 5 postoperativen Tage erzielten die mit kortisongetränktem Bindegewebe Behandelten einer anderen Studiengruppe einen mittleren Hörerfolg von $31 \mathrm{~dB}$ auf einen Endwert von $42 \mathrm{~dB}$. Die Vergleichswerte in der Gruppe der Erkrankten, die kein vorbehandeltes Bindgewebe implantiert bekamen, betrugen $15 \mathrm{~dB}$ Hörgewinn mit einem durchschnittlichen erreichten „Endhörverlust“ (innerhalb von 5 Tagen) von $58 \mathrm{~dB}$. Demgegenüber konnten Patienten unseres Kollektives innerhalb dieser kurzen Zeit nach dem chirurgischen Eingriff nur einen Hörgewinn von 2,1 dB auf einen Hörverlustwert von 97,2 dB erzielen. Damit war bei unseren Patienten innerhalb der ersten 5 Tage nach Operation nur $6,5 \%$ des Gesamthörerfolges erreicht worden.

Bei Verwendung der modifizierten Kanzaki-Kriterien konnten bei den Typen 1-3 (Hörerholung $>10 \mathrm{~dB}$ ) in drei Studien mit Einbringung von kortisongetränktem Bindegewebe Hörverbesserungen von 52\%, $69 \%$ resp. $83 \%$ beobachtet werden. Demgegenüber erzielten die Gruppen, bei denen kein vorbehandeltes Bindegewebe in die Pauke eingebracht wurde, prozentuale Hörverbesserungsraten von 32,3\% (eigenes Patientengut) resp. $63 \%$ (publizierte Studiengruppe).

Die zeitliche Hörerholung bei Patienten, die intratympanale Kortikoidinjektionen erhalten hatten, trat im Durchschnitt unmittelbar nach Abschluss der meist 2-wöchigen Therapie auf $(n=436)$, während sie sich bei Patienten mit Tympanoskopie $(n=31)$ erst mit einer Verzögerung von einigen Tagen einstellte. 
Dabei ist zu berücksichtigen, dass bei der erstgenannten Patientengruppe bei Therapieabschluss bereits ein 14-tägiges Zeitintervall nach der ersten Applikation der Steroide in die Pauke vorlag.

Bei dem Vergleich der zeitlichen Entwicklung der relativen Hörverbesserungen berichteten 2 Veröffentlichungen desselben Hauptautors von einem ähnlichen Verlauf nach Tympanoplastik mit Einbringen von kortisongetränktem Bindgewebe wie nach intratympanalen Kortikoidinjektionen. Bei einem anderen Autor glich der zeitliche Hörkurvenverlauf mit dem Eintreten der Hörverbesserung dem, wie er sonst nach Tympanoplastik mit Einbringung von unbehandeltem Bindgewebe beobachtet worden war.

Die Datenlage zur Bewertung der Erfolge der Implantation von kortisongetränktem Bindegewebe ist noch sehr gering, von unterschiedlichen Auswertungskriterien gekennzeichnet und zeigt bislang widersprüchliche Ergebnisse. Dies betrifft zum einen die Angabe der maximal mit dieser Methode erreichbaren Hörerfolge, zum anderen den Zeitverlauf des Eintritts der Hörverbesserung. Es ist zu erwarten, dass in der Zukunft mehr Studien erscheinen, die diesen relativ neuen Weg (Obliteration mit autologem Bindegewebe) einschlagen werden.

Open Access Dieses Kapitel wird unter der Creative Commons Namensnennung 4.0 International Lizenz (http://creativecommons.org/licenses/by/4.0/deed.de) veröffentlicht, welche die Nutzung, Vervielfältigung, Bearbeitung, Verbreitung und Wiedergabe in jeglichem Medium und Format erlaubt, sofern Sie den/die ursprünglichen Autor(en) und die Quelle ordnungsgemäß nennen, einen Link zur Creative Commons Lizenz beifügen und angeben, ob Änderungen vorgenommen wurden.

Die in diesem Kapitel enthaltenen Bilder und sonstiges Drittmaterial unterliegen ebenfalls der genannten Creative Commons Lizenz, sofern sich aus der Abbildungslegende nichts anderes ergibt. Sofern das betreffende Material nicht unter der genannten Creative Commons Lizenz steht und die betreffende Handlung nicht nach gesetzlichen Vorschriften erlaubt ist, ist für die oben aufgeführten Weiterverwendungen des Materials die Einwilligung des jeweiligen Rechteinhabers einzuholen.

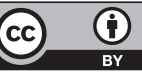

